\title{
Which of My Transient Type Checks Are Not (Almost) Free?
}

\author{
Isaac Oscar Gariano \\ Engineering and Computer Science \\ Victoria University of Wellington \\ New Zealand \\ Isaac@ecs.vuw.ac.nz
}

\author{
Richard Roberts \\ Computational Media Innovation \\ Centre \\ Victoria University of Wellington \\ New Zealand \\ rykardo.r@gmail.com
}

\author{
Stefan Marr \\ School of Computing \\ University of Kent \\ United Kingdom \\ s.marr@kent.ac.uk
}

\author{
Michael Homer \\ Engineering and Computer Science \\ Victoria University of Wellington \\ New Zealand \\ mwh@ecs.vuw.ac.nz
}

\author{
James Noble \\ Engineering and Computer Science \\ Victoria University of Wellington \\ New Zealand \\ kjx@ecs.vuw.ac.nz
}

\begin{abstract}
One form of type checking used in gradually typed language is transient type checking: whenever an object 'flows' through code with a type annotation, the object is dynamically checked to ensure it has the methods required by the annotation. Just-in-time compilation and optimisation in virtual machines can eliminate much of the overhead of runtime transient type checks. Unfortunately this optimisation is not uniform: some type checks will significantly decrease, or even increase, a program's performance.

In this paper, we refine the so called "Takikawa" proto$\mathrm{col}$, and use it to identify which type annotations have the greatest effects on performance. In particular, we show how graphing the performance of such benchmarks when varying which type annotations are present in the source code can be used to discern potential patterns in performance. We demonstrate our approach by testing the Moth virtual machine: for many of the benchmarks where Moth's transient type checking impacts performance, we have been able to identify one or two specific type annotations that are the likely cause. Without these type annotations, the performance impact of transient type checking becomes negligible.

Using our technique programmers can optimise programs by removing expensive type checks, and VM engineers can identify new opportunities for compiler optimisation.
\end{abstract}

VMIL '19, October 22, 2019, Athens, Greece

(c) 2019 Copyright held by the owner/author(s). Publication rights licensed to ACM.

This is the author's version of the work. It is posted here for your personal use. Not for redistribution. The definitive Version of Record was published in Proceedings of the 11th ACM SIGPLAN International Workshop on Virtual Machines and Intermediate Languages (VMIL '19), October 22, 2019, Athens, Greece, https://doi.org/10.1145/3358504.3361232.
CCS Concepts • Software and its engineering $\rightarrow$ Software performance; Object oriented languages; fust-in-time compilers.

Keywords dynamic type checking, gradual types, optional types, Grace, performance evaluation, benchmarking, objectoriented programming

ACM Reference Format:

Isaac Oscar Gariano, Richard Roberts, Stefan Marr, Michael Homer, and James Noble. 2019. Which of My Transient Type Checks Are Not (Almost) Free?. In Proceedings of the 11th ACM SIGPLAN International Workshop on Virtual Machines and Intermediate Languages (VMIL '19), October 22, 2019, Athens, Greece. ACM, New York, NY, USA, 9 pages. https://doi.org/10.1145/3358504.3361232

\section{Introduction}

Gradual typing aims to add static type annotations to dynamic languages, increasing their safety while maintaining flexibility $[8,31,33]$, and/or, permitting dynamic type annotations within static languages, increasing flexibility whilst maintaining some safety [1].

There is a vast spectrum of different approaches to gradual typing $[10,14]$. Here we measure the performance of "transient" or "type-tag" checks (as in Reticulated Python), which offer first-order semantics: they check that an object's type constructor or names of supported methods match any static types that the object flows through, but not the return types or argument types of those methods [7, 15, 28, 32, 37].

Unfortunately most gradual systems with run-time semantics, as opposed to type erasure as in TypeScript, impose significant run-time performance overheads. This has lead to a significant body of research to develop techniques to optimise gradual typing $[3,15,25,27,39]$.

This has also lead to a technique to evaluate the performance of gradual typing, the "Takikawa" protocol [16, 36, 38]. The Takikawa protocol was created to measure the run-time cost of gradual typing by testing various configurations of 
typed and untyped code. This approach was designed to characterise how the amount of typed and untyped code influences performance. In particular, Takikawa protocol evaluations often show that simply adding more type annotations does not always produce a uniform effect on performance. Here we adapt their approach in order to identify individual type annotations that may be responsible for performance effects.

This paper builds upon our recent work on optimising transient type checks $[29,30]$ to make the following contributions:

- an approach to identifying individual gradual type annotations that cause significant performance effects

- an observation that the overhead of Moth's transient type checking on small benchmarks (with 10-250 type annotations) is usually caused by only one or two type annotations

The next section discusses dynamic type checks and gradual typing in Moth (an implementation of the Grace language), then section 3 describes our benchmarking protocol. Section 4 then presents the overall results of our benchmarks, while section 5 looks at the results of benchmarking individual type checks. Section 6 presents some additional related work, and finally 7 summarises our results, and briefly considers threats to validity.

\section{Background}

Our work is based on the Moth virtual machine [29, 30], an implementation of the Grace programming language [5, 9]. Moth is based on the Graal and Truffle toolchain [41, 42], and developed from a Newspeak implementation based on the Simple Object Machine [12, 23].

\subsection{Grace and Transient Type Checking}

Grace is an object-oriented, imperative, educational programming language, with a focus on introductory programming courses, but also intended for more advanced study and research $[5,9]$. While Grace's syntax draws from the so-called "curly bracket" traditions of C, Java, and JavaScript, the structure of the language is in many ways closer to Smalltalk: all computation is done via dynamically dispatched "method requests" where the object receiving the request decides what code to run, control structures are built out of lambda expressions support "non-local" returns, i.e. they can return to the point where execution first encountered the lambda [13]. In other ways, Grace is closer to JavaScript than Smalltalk: Grace objects are created from object literals, rather than by instantiating classes $[6,20]$ and objects and classes can be deeply nested within each other [21].

Grace's Typing In Grace, all declarations can be annotated with types. As Grace is designed to support a variety of teaching methods, implementation of Grace are free to check such type annotations statically, dynamically, or not at all. The type system of Grace is intrinsically gradual: type annotations should not affect the semantics of a correct program [33]. The type system includes a distinguished "Unknown" type which matches any other type; this unknown type is the default when type annotations are omitted.

Static typing for the core of Grace's type system has been described elsewhere [19]; here we explain how these types can be understood dynamically, from the Grace programmer's point of view. Grace's types are structural [5], that is, an object conforms to a type whenever it conforms to the "structural" requirements of a type, rather than requiring classes or objects to explicitly declare their intended type.

In Grace, types specify a set of method signatures that an object must provide. A type expresses the requests an object can respond to, for example whether a particular accessor is available, rather than a location in a class hierarchy.

Moth's Transient Type Checking Moth's implementation of transient type checks are only only first-order. Moth only checks dynamically that an object has methods of the same name and arity as are required by a type: any argument and return types of such methods are not checked.

In particular, Moth performs the following type checks at run time:

- when a method is requested, arguments that are passed are checked against the corresponding parameter type annotations of the called method, this is done before the body of the method is executed;

- when the body of a method has finished executing, but before it returns to its caller, the method's return value is checked against the return type annotation of the called method;

- whenever a variable is read or written to, its value is checked against the type specified by the variables declaration.

To see how this works in practice, consider this piece of Grace code:

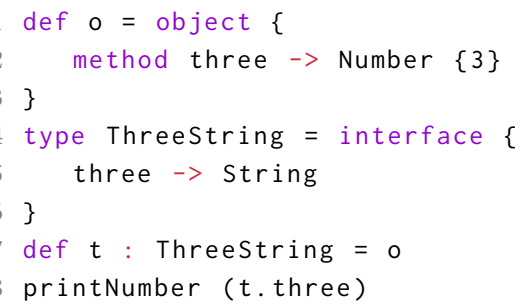

Moth will perform dynamic type checks:

- on line 7, when the o object initialises the variable $t$, Moth checks that o has a 0 -argument method called three;

- on line 8 , when the value of $t$ is read, Moth checks that its value (o) still has a three method; 
- on line 2, when the method requested by " $t$. three" returns, Moth checks that returned value conforms to the Number type; and (presumably) within the definition of printNumber ( $\mathrm{n}$ : Number) (not shown), Moth will again check that the value is a Number.

Note that we never check whether the result of requesting "t. three" is actually a String (as one may expect from line 5) because Moth only performs first-order type checks (it checks whether objects have conforming methods) not higher-order checks (whether the argument and result types of methods' conform). In addition, Moth only checks when values flow through explicit type annotations. This is why the type declared in lines 4-6 is checked only on line 7 (where it is mentioned explicitly); and the check only requires the presence of a method called three, regardless of the method's declared return type.

Moth's Optimisation We are developing Moth as a research platform [30]. Like other VMs based on the Truffle and Graal toolchain, Moth is a self-optimising AST interpreter [43]. The key idea is that an AST rewrites itself based on a program's run time values to reflect the minimal set of operations needed to execute the program correctly. The rewritten AST is then compiled into efficient machine code. This rewriting often depends on the dynamic types of the objects involved. In the simplest case, a "self" call (when one method on an object requests a second method on the exact same object) will always result in executing the exact same method. Thus the called method can be inlined into the callee, avoiding overhead of a machine-level subroutine invocation and an object-oriented dynamic dispatch.

Moth relies on a number of standard techniques for optimising object-oriented programs. "Shapes" [40] capture information about objects' structures and (run time) field types, allowing a just-in-time compiler to represent objects in memory similarly to $\mathrm{C}$ structs and, consequently, can generate highly efficient code. "Polymorphic inline caches" [17] use object shapes to cache the results of method lookups, avoiding expensive class hierarchy searches or indirect jumps through virtual method tables. Since Moth is built on the Truffle framework, Graal comes with additional support for partial evaluation, which enables efficient native code generation for Truffle interpreters [41].

\section{Experimental Methodology}

Our goal is to identify which type annotations in Grace programs cause performance effects. To this end, we built upon the so-called "Takikawa" or "Takikawa-Greenman" evaluation protocol $[16,36]$. It uses $2^{N}$ configurations of each benchmark. A configuration is a particular mix of static and dynamically typed code, forming a lattice of configurations. We only test a relatively small sample of this lattice, which is in our experience sufficient to pinpoint performance anomalies caused by type annotations.

\subsection{The Takikawa Protocol}

The Takikawa evaluation protocol was originally proposed for Typed Racket, where static vs dynamic typing is set per-module, so $N$ is the number of modules. The original Takikawa protocol also suggested a sampling strategy, where $10 N$ configurations are randomly chosen from the lattice, however the lattice is a binomial distribution, meaning the majority of chosen benchmarks will have around $N / 2$ type annotations.

Grace allows programmers to choose whether each individual declaration should be type-checked, and thus follows languages such as Reticulated Python [34, 37, 39]. This means in Grace $N$ is the number of type annotations in the program, so it is infeasible to check an entire lattice, even for a moderately sized benchmark. Vitousek et al. therefore modified the Takikawa protocol for these kinds of languages by using a different form of sampling [38]. The TakikawaVitousek protocol divides the number of type annotations in a fully-typed program into a maximum of 100 intervals, and then randomly generates ten programs within each interval by erasing type annotations. However, this approach was designed for benchmarks with large numbers of type annotations, as well as for a larger sample size than our work.

Refined Takikawa Protocol. Unlike prior work, we wish to identify which type annotations cause anomalies, and thus we adapted the Takikawa protocol and took inspiration from the Takikawa-Vitousek variant. For each benchmark, we generated 100 partially typed versions, or fewer if the benchmark has less than 11 types. We did an even split so that for each $i \geq 1$ and $i<N$, we generated roughly the same number of configurations with $i$ type annotations. We used Robert Floyd's sampling algorithm [4] to randomly choose the type annotations each configuration contained, and we ensured that no duplicate configurations were generated. In addition to these, we tested fully untyped and typed versions, for a total of 102 configurations per benchmark (or 97 in the case of our Storage benchmark, since it only has 10 type annotations).

\subsection{The Benchmarks}

For this work, we rely on the benchmark suite compiled for previous work [30]. It is a collection of 21 benchmarks in total, derived from the Are We Fast Yet benchmark suite [24] and other benchmarks from the gradual-typing literature.

We added type annotations to every benchmark, aiming to use the most appropriate (i.e. most specific) annotation for each declaration.

In our previous work, we [30] we determined that the overhead of type checking on Moth is on average of $5 \%$ (min. $-13 \%$, max. $79 \%$ ). This compares the peak performance of Moth with all checks disabled against an execution that has all checks enabled. 


\subsection{Experimental Set Up}

To account for the complex warmup behaviour of modern systems [2] as well as the non-determinism caused by e.g. garbage collection and cache effects, we ran each benchmark for 1000 iterations in the same invocation of Moth, and discard the first 350 iterations to ignore warmup JIT compilation. Our previous work identified this as a suitable cut off [30].

Though outliers remain visible in the plots for each individual benchmark, the largest $95 \%$ confidence interval we obtained (over the mean time after warmup) for any of experiments was $\pm 8.3 \%$ (for the PyStone benchmark).

All our experiments used the same machine, Graal, and Moth as previously; the machine has two Intel Xeon E5-2620 v3 $2.40 \mathrm{GHz}$, with 6 cores each, for a total of 24 hyperthreads. The machine was running Ubuntu Linux 16.04.6, with Kernel 4.4, and we used ReBench 1.0 [22] and Java 1.8.0_191 Graal 0.43 . Benchmarks were executed one by one to avoid interference between them. The analysis of the results and plots where generated using Python 3.7.3 and PGFPLOTS 1.16. To enable reproductions, the scripts we used to generate and run our experiments, including the source code for all the configurations tested, are available online. ${ }^{1}$

In our previous work [30], we also compared the performance of untyped code on Moth against state-of-the-art VMs: Java, Node.js using the V8 JavaScript VM, and the Higgs JavaScript VM. Java was the fastest of these, and on average V8 was about $1.8 \mathrm{x}$ slower than Java, Moth was $2.3 \mathrm{x}$ slower, and Higgs was $10.4 \mathrm{x}$ slower. We believe this makes Moth suitable for assessing the impact of type checking, because Moth's performance is close enough to state-of-the-art VMs, which should make it harder to hide type checking overheads in a slow baseline.

\section{Performance of Benchmark Configurations}

Before we start to investigate specific type annotations, we present the performance measurements of our sample of the typing lattice configurations in figure 1 . These results are the foundation for a more detailed analysis.

Following [38], the points on each graph in figure 1 show the average execution of each individual configuration. The $\mathrm{x}$-axis represents the proportion of type annotations for each configuration, with the left- and right-most points showing the times for the fully untyped and typed configurations respectively. The execution time in milliseconds is shown on the left $y$-axes, and time relative to the fully untyped configuration is shown on the right y-axes.

Most of the graphs are essentially horizontal lines, indicating that the overhead of including type annotations is negligible. The plots for CD and Richards show a roughly linear

\footnotetext{
${ }^{1}$ https://gitlab.ecs.vuw.ac.nz/isaac/Moth-Takikawa
}

increase, i.e. for these two benchmarks, adding type annotations reduces performance linearly. On the other hand, the plots for Go, Permute, DeltaBlue, and Storage show decreases: i.e. adding more type annotations improves performance of these benchmarks.

By inspecting the scatterplots, we observe that the performance of Moth in almost any configuration of a benchmark is bounded by the performance in the untyped and fully-typed configuration. That is, for these benchmarks on transient type checks, measuring just the untyped and fullytyped configurations would provide excellent estimates of a benchmark's performance bounds. This is different to the experience of other kinds of gradual typing, where the best, and most importantly worst, configurations are not always those fully typed or fully untyped [16]. However, the Richards benchmark does have sections outside these bounds, and isolated executions of a couple of others (Fannkuch and DeltaBlue) are also outliers. We believe that the $0 \%$ and $100 \%$ bounds are nonetheless a reasonable heuristic estimator in most cases, and we will examine the outlying benchmarks further.

Of particular note is that some of the graphs (Permute, Storage, Towers, and Richards) show bimodal performance profiles, that is, two separate roughly-horizontal lines. Presumably Moth can remove all the overhead from some configurations, but in others there must be one or more type checks that could not be optimised away. List shows three performance modes: the graph consists of three mostly flat lines, at about 1 times slower, 1.5 times slower, and 1.8 times slower than untyped code.

\section{Identifying Type Annotations With Signification Performance Impact}

We hypothesise that the previously identified bi/tri-modal performance behaviour as seen in the graphs for Permute and others (cf. figure 1) are caused by only a few type annotations: i.e. there are a very few annotations that determine each benchmark's performance.

To verify this hypothesis for each type annotation we measured one additional configuration, with only that single type annotation present. We did this to compare the overhead of each type annotation in isolation against the no-typecheck baseline.

Figure 2 shows the results of these experiments. It shows a pair of graphs for a selection of ten benchmarks: it's associated typing lattice scatter plot and a column graph showing the results of these single type annotation experiments.

The column graphs, to the right of the corresponding scatter plot, show the execution time of configurations with only a single type annotation, the $\mathrm{x}$-axis indicates the index of this annotation, thus the first column represents the first annotation, and the last column represents the last one (in 
Bounce (22 Type Annotations)

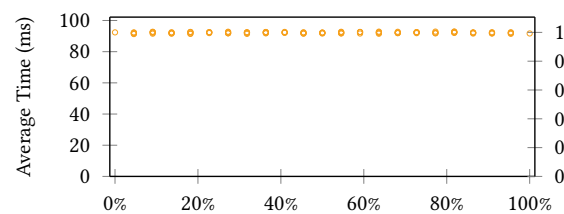

Fannkuch (27 Type Annotations)

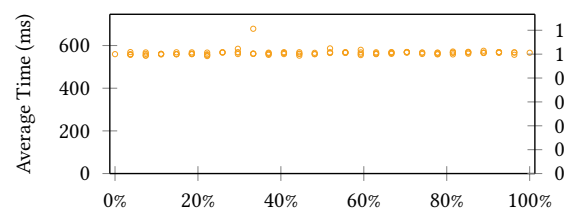

GraphSearch (58 Type Annotations)

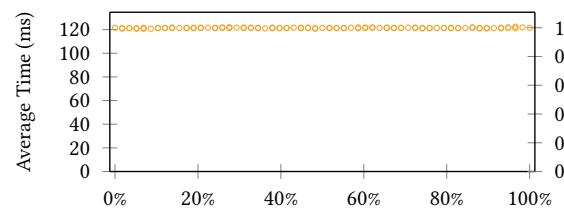

List (23 Type Annotations)

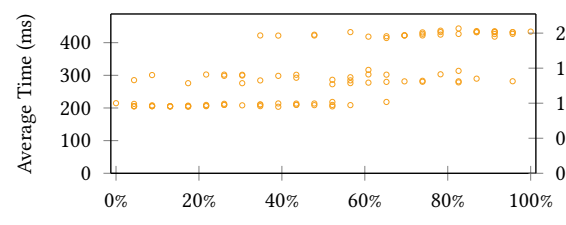

Permute (14 Type Annotations)

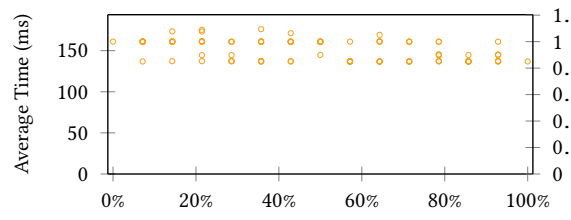

Richards (177 Type Annotations)

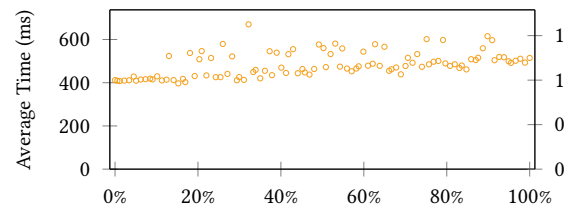

SpectralNorm (39 Type Annotations)

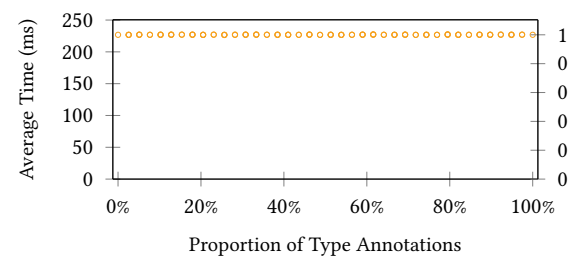

Proportion of Type Annotations
CD (250 Type Annotations)

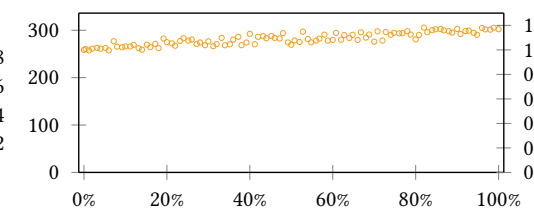

Float (30 Type Annotations)

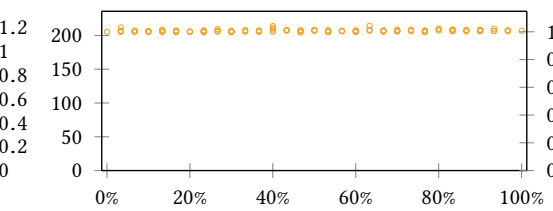

Havlak (210 Type Annotations)

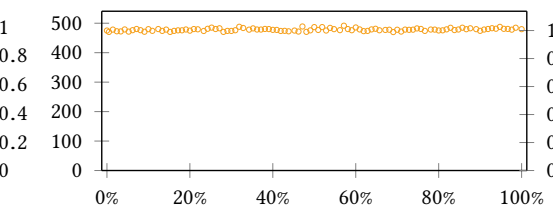

Mandelbrot (23 Type Annotations)

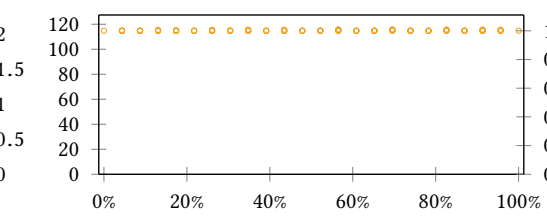

PyStone (85 Type Annotations)

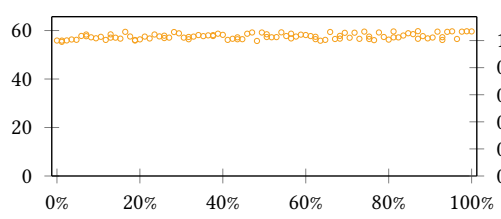

Sieve (13 Type Annotations)

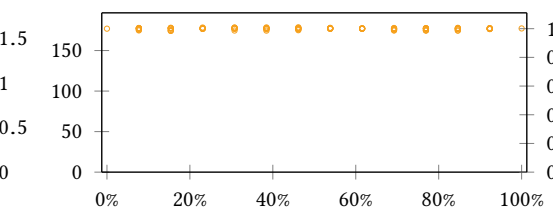

Storage (10 Type Annotations)

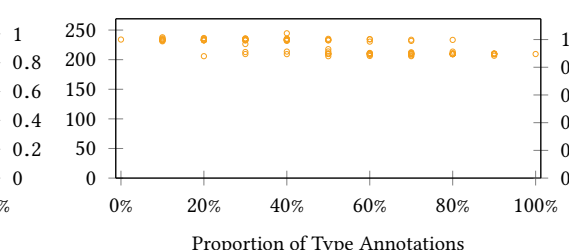

Proportion of Type Annotations
DeltaBlue (243 Type Annotations)



Go (230 Type Annotations)

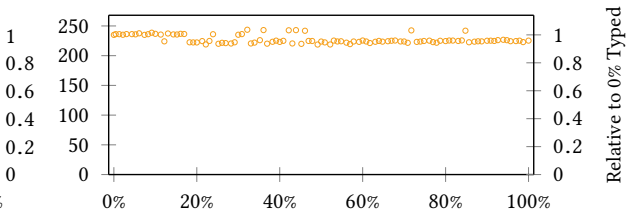

Json (134 Type Annotations)

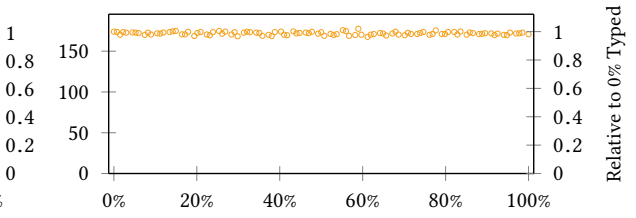

NBody (65 Type Annotations)

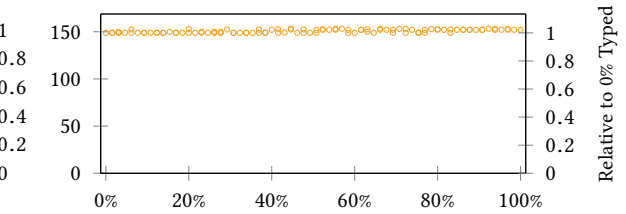

Queens (22 Type Annotations)

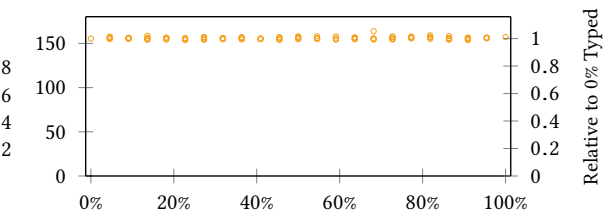

Snake (70 Type Annotations)

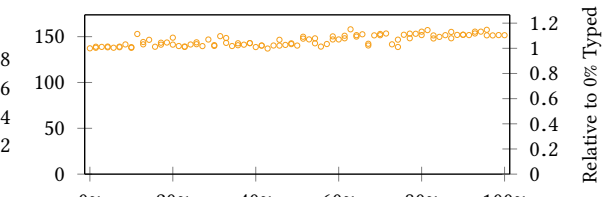

Towers (30 Type Annotations)

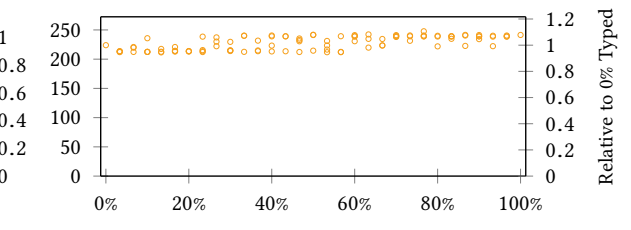

Proportion of Type Annotations

Figure 1. Graphs of (at most) 102 configurations in the typing lattices for each benchmark. Time is measured as the mean of the $351^{\text {st }}$ to the $1,000^{\text {th }}$ benchmark iteration under a single invocation of Moth (lower is better). 
Snake

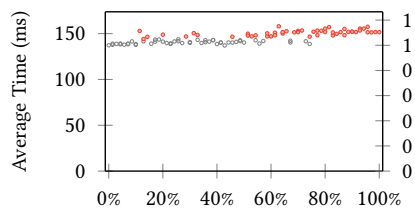

Towers

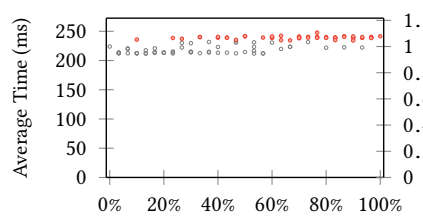

DeltaBlue

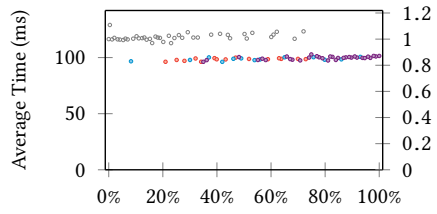

List
(70 Type Annotations)

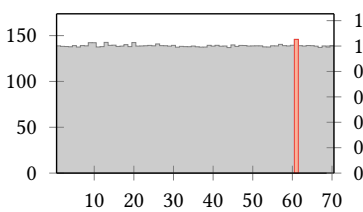

(30 Type Annotations)

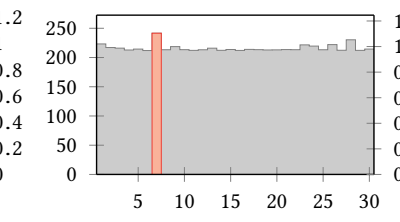

(243 Type Annotations)

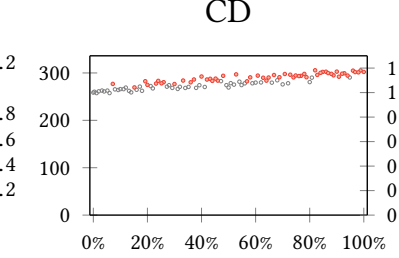

Permute



Go

(250 Type Annotations)

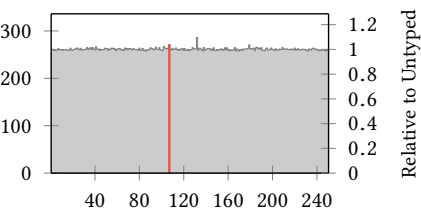

(14 Type Annotations)

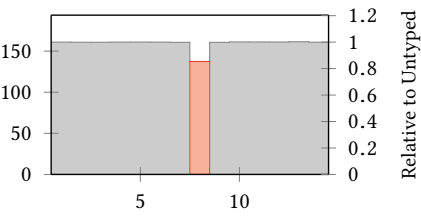

(230 Type Annotations)

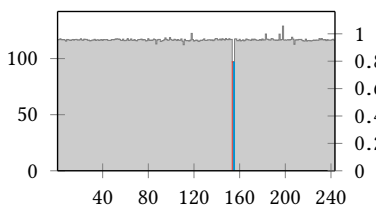

(23 Type Annotations)

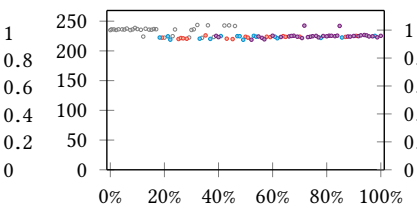

Richards

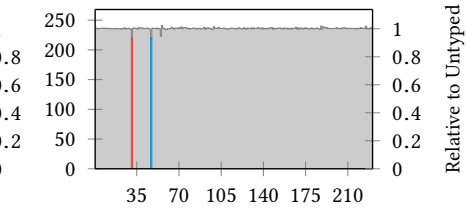

(177 Type Annotations)

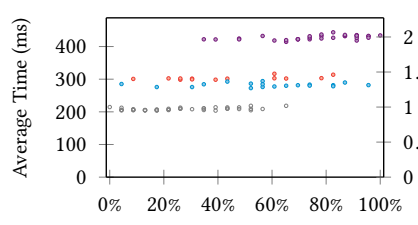

Json

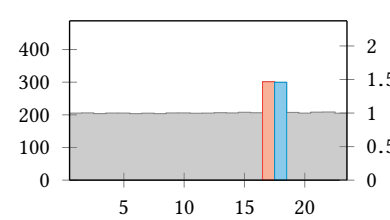

(134 Type Annotations)

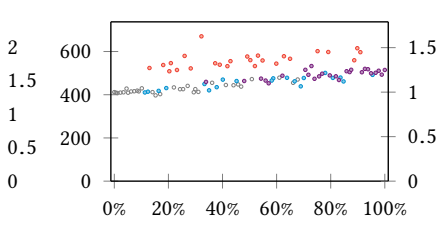

PyStone

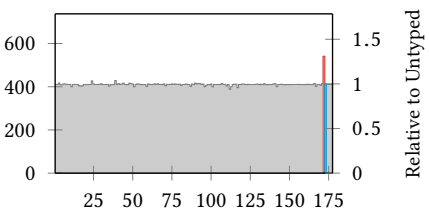

(85 Type Annotations)

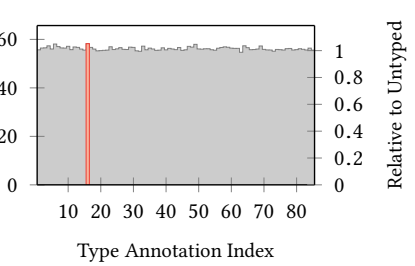

Figure 2. Pairs of colour coded scatter and column graphs. The scatter graphs represent the performance of a sample of the typing lattices. The column graphs show the performance of every configuration with only one type annotation. The scatter plots and column graphs are colour coded based on whether a particular type annotation or two are present in the source code.

the order they appear in the source code). The y-axes for the column graphs are the same as the associated scatter graphs.

For each benchmark we highlighted one or two type annotations that seem to show a pattern for the typing lattice performance scatter plots, or in the case of SpectralNorm and Json had higher than usual columns. The identified types are those represented by the red and blue columns. The scatter plots are colour-coded accordingly: a red or blue circle represents configurations with the given type annotation present, but not both, purple circles represent configurations with both type annotations present, and grey circles represent those with neither. Though we exhaustively inspected such colour coded scatter plots for all 1,775 type annotations across all 21 benchmarks, the only patterns we noticed are those shown in 2.

As can be seen, most of the patterns we found in the typing lattice graphs correspond to outliers in the single type annotation column graphs. For the Snake, Towers and CD benchmarks, there is clearly a pattern where an individual type annotation (highlighted in red) appears responsible for the upper/slowest half of the typing lattice graphs. For Permute there is actually a significant performance increase, indicated by the lower half of the lattice being highlighted in red. DeltaBlue and Go also show a slight increase in performance, but here this is caused by two type annotations (in red and blue); this effect is not cumulative, however: the 
purple dots are about the same height as the red and blue ones, so either annotation is sufficient for the full benefit.

List is interesting as it demonstrates that the red and blue type annotations both cause a significant decrease in performance, which is even greater when both are present. Richards is particularly odd as it shows a performance decrease that occurs when the red type annotation is present, but not the blue one. We had previously identified this benchmark and the aforementioned type annotations [30] as being the worst case for Moth.

For Json and PyStone, although we found outliers in the column graphs (such as types \#112 and \#16, highlighted in red), we were unable to find a matching pattern in the configuration performance. Finally, for DeltaBlue there is a noticeable spike in the column graphs (at \#198), however we again found no apparent relation between this outlier and the overall performance.

In particular, observe that the grey dots for Snake, Towers, DeltaBlue, Go, and List are mostly flat horizontal lines, this indicates that by simply deleting the red and blue type annotations, the performance impact of transient type checking becomes negligible. However for CD and Richards, the transient type checking overhead of the grey dots is roughly linear, albeit still less than with the red and blue type annotations present. Thus we can observe that usually, only a couple of type annotations are responsible for the overhead caused by Moth's transient typed checking, whereas the rest are "free".

The remaining benchmarks, which we have not shown in figure 2, have even flatter column graphs than PyStone, and we could not identify any patterns in the typing lattices. Of those we did not show, the greatest difference in single-type configurations relative to the untyped configuration was $5.07 \%$ (for the Float benchmark).

\section{Related Work}

The high-performance computing community has been investigating how tools and visualisations can help developers to utilise their systems more efficiently $[11,26]$. Their focus is typically on parallelisation opportunities, guided by runtime feedback, cost models, or heuristics. Their large body of work [18] uses various approaches, though, we are not aware of work that has used an approach similar to ours.

At the moment, our approach to identifying type annotations that cause performance anomalies is not integrated into a development environment. Though, for instance Optimization Coaching [35] is a promising direction. Optimization Coaching uses feedback from the runtime to guide developers to insert or change type declarations to enable a compiler to generate a more optimal program. In this spirit, we would eventually want to achieve the same, although in our case, we need to run full experiments to get the necessary information.

\section{Discussion and Conclusion}

In this paper we have investigated how benchmarks can be repurposed to determine precisely which transient type checks are likely to cause performance effects, and are currently not optimised away by the just-in-time compiler. However, detailed analysis will be needed in order to identify exactly what causes such performance effects: we previously undertook such an analysis for the List benchmark [30].

We observed that many of our benchmark results conducted under the Takikawa protocol showed a characteristic bimodal performance profile: some of the benchmark configurations ran significantly slower than the remaining configurations. We also observed trimodal profiles, as well as performance increases when type annotations where added.

By inspecting graphs of the performance of where only one type annotation is present, we can easily identify type annotations that likely have a significant effect on performance. By then inspecting the typing lattice graphs colour coded based on whether such type annotation was present or not, we were often able to notice patterns. In particular, these patterns suggested the just one or two type annotations are likely responsible for the bimodal performance and most of the overhead caused by Moth's transient type checking. Though every type annotation that showed a significant effect across the typing lattice also showed a significant performance effect when no other typing annotations where present, the converse did not hold.

This is preliminary work, in particular we have not yet identified exactly why these type annotations show an effect on performance. There are also a number of threats to validity. Regarding construct validity, our underlying implementation may contain undetected bugs that affect the semantics or performance of the gradual typing checks. Regarding internal validity, our benchmarking harness runs on the same implementation and therefore is subject to the same issues. Regarding external validity, Moth is built on the Truffle and Graal toolchain, so we expect to resemble other Graal VMs doing similar AST-based optimizations of transient type checks. Because we rely on common techniques, we expect our results to be transferable to other JIT implementations as well.

Finally, it is not clear how our results would transfer to other gradually typed-languages or other semantics for gradual typing. Our benchmarks do not depend on any features of Grace that are not common in other object-oriented languages, but as Grace lacks a large corpus of programs the benchmarks are necessarily small and artificial. The advantage of Grace for this research is that their relative simplicity means we have been able to build an implementation that features competitive performance with significantly less effort than would be required for larger and more complex languages. 
In the future, we hope to investigate statistical techniques to determine the significance of each type annotation's contribution to a programs overall performance. We would also like to investigate whether this approach can assist with optimisations for programmers' day-to-day development, or help VM engineers identifying performance bugs in the underlying virtual machines.

\section{Acknowledgments}

The authors would like to thank the anonymous reviewers for their valuable comments and helpful suggestions. This work is supported in part by the Royal Society of New Zealand (Te Apārangi) Marsden Fund (Te Pūtea Rangahau a Marsden) under grant VUW1815.

\section{References}

[1] Martín Abadi, Luca Cardelli, Benjamin C. Pierce, and Gordon D. Plotkin. 1991. Dynamic Typing in a Statically Typed Language. ACM Trans. Program. Lang. Syst. 13, 2 (1991), 237-268.

[2] Edd Barrett, Carl Friedrich Bolz-Tereick, Rebecca Killick, Sarah Mount, and Laurence Tratt. 2017. Virtual Machine Warmup Blows Hot and Cold. Proc. ACM Program. Lang. 1, OOPSLA, Article 52 (Oct. 2017), 27 pages.

[3] Spenser Bauman, Carl Friedrich Bolz-Tereick, Jeremy Siek, and Sam Tobin-Hochstadt. 2017. Sound Gradual Typing: Only Mostly Dead. Proc. ACM Program. Lang. 1, OOPSLA, Article 54 (Oct. 2017), 24 pages.

[4] Jon Bentley and Bob Floyd. 1987. Programming Pearls: A Sample of Brilliance. Commun. ACM 30, 9 (Sept. 1987), 754-757. https: //doi.org/10.1145/30401.315746

[5] Andrew P. Black, Kim B. Bruce, Michael Homer, and James Noble. 2012. Grace: the absence of (inessential) difficulty. In Onward! '12: Proceedings 12th SIGPLAN Symp. on New Ideas in Programming and Reflections on Software. ACM, New York, NY, 85-98.

[6] Andrew P. Black, Norman C. Hutchinson, Eric Jul, and Henry M. Levy 2007. The development of the Emerald programming language. In Proceedings of the Third ACM SIGPLAN History of Programming Languages Conference (HOPL-III), San Diego, California, USA, 9-10 June 2007. 1-51.

[7] Bard Bloom, John Field, Nathaniel Nystrom, Johan Östlund, Gregor Richards, Rok Strniša, Jan Vitek, and Tobias Wrigstad. 2009. Thorn: Robust, Concurrent, Extensible Scripting on the JVM. In Proceedings of the ACM SIGPLAN-SIGACT Symposium on Principles of Programming Languages (POPL). 117-136.

[8] Gilad Bracha. 2004. Pluggable Type Systems. OOPSLA Workshop on Revival of Dynamic Languages. , 6 pages.

[9] Kim Bruce, Andrew Black, Michael Homer, James Noble, Amy Ruskin, and Richard Yannow. 2013. Seeking Grace: a new object-oriented language for novices. In Proceedings 44th SIGCSE Technical Symposium on Computer Science Education. ACM, 129-134.

[10] Benjamin Chung, Paley Li, Francesco Zappa Nardelli, and Jan Vitek. 2018. KafKa: Gradual Typing for Objects. In 32nd European Conference on Object-Oriented Programming, ECOOP 2018, fuly 16-21, 2018, Amsterdam, The Netherlands. 12:1-12:24. https://doi.org/10.4230/LIPIcs. ECOOP.2018.12

[11] Anderson B. N. da Silva, Daniel A. M. Cunha, Vitor R. G. Silva, Alex F. de A. Furtunato, and Samuel Xavier-de Souza. 2019. PaScal Viewer: A Tool for the Visualization of Parallel Scalability Trends. In Programming and Performance Visualization Tools, Abhinav Bhatele, David Boehme, Joshua A. Levine, Allen D. Malony, and Martin Schulz (Eds.). Springer International Publishing, Cham, 250-264.
[12] Benoit Daloze, Stefan Marr, Daniele Bonetta, and Hanspeter Mössenböck. 2016. Efficient and Thread-Safe Objects for Dynamically-Typed Languages. In Proceedings of the 2016 ACM International Conference on Object Oriented Programming Systems Languages \& Applications (OOPSLA'16). ACM, 642-659.

[13] Adele Goldberg and David Robson. 1983. Smalltalk-80: The Language and its Implementation. Addison-Wesley.

[14] Ben Greenman and Matthias Felleisen. 2018. A spectrum of type soundness and performance. PACMPL 2, ICFP (2018), 71:1-71:32. https: //doi.org/10.1145/3236766

[15] Ben Greenman and Zeina Migeed. 2018. On the Cost of Type-Tag Soundness. In Proceedings of the ACM SIGPLAN Workshop on Partial Evaluation and Program Manipulation (PEPM'18). ACM, 30-39.

[16] Ben Greenman, Asumu Takikawa, Max S. New, Daniel Feltey, Robert Bruce Findler, Jan Vitek, and Matthias Felleisen. 2019. How to evaluate the performance of gradual type systems. fournal of Functional Programming 29 (2019), $45 . \quad$ https://doi.org/10.1017/ S0956796818000217

[17] Urs Hölzle, Craig Chambers, and David Ungar. 1991. Optimizing Dynamically-Typed Object-Oriented Languages With Polymorphic Inline Caches. In ECOOP '91: European Conference on Object-Oriented Programming (LNCS), Vol. 512. Springer, 21-38. https://doi.org/10. 1007/BFb0057013

[18] Katherine E. Isaacs, Alfredo Giménez, Ilir Jusufi, Todd Gamblin, Abhinav Bhatele, Martin Schulz, Bernd Hamann, and Peer-Timo Bremer. 2014. State of the Art of Performance Visualization. In EuroVis - STARs, R. Borgo, R. Maciejewski, and I. Viola (Eds.). The Eurographics Association. https://doi.org/10.2312/eurovisstar.20141177

[19] Timothy Jones. 2017. Classless Object Semantics. Ph.D. Dissertation. Victoria University of Wellington.

[20] Timothy Jones, Michael Homer, James Noble, and Kim Bruce. 2016. Object Inheritance Without Classes. In 30th European Conference on Object-Oriented Programming (ECOOP 2016), Vol. 56. 13:1-13:26.

[21] Ole Lehrmann Madsen, Birger Møller-Pedersen, and Kristen Nygaard. 1993. Object-Oriented Programming in the BETA Programming Language. Addison-Wesley.

[22] Stefan Marr. 2018. ReBench: Execute and Document Benchmarks Reproducibly. https://doi.org/10.5281/zenodo.1311762 Version 1.0.

[23] Stefan Marr. 2018. SOMns: A Newspeak for Concurrency Research. https://doi.org/10.5281/zenodo.3270908

[24] Stefan Marr, Benoit Daloze, and Hanspeter Mössenböck. 2016. CrossLanguage Compiler Benchmarking-Are We Fast Yet?. In Proceedings of the 12th Symposium on Dynamic Languages (DLS'16). ACM, 120-131.

[25] Fabian Muehlboeck and Ross Tate. 2017. Sound Gradual Typing is Nominally Alive and Well. Proc. ACM Program. Lang. 1, OOPSLA, Article 56 (Oct. 2017), 30 pages.

[26] E. Papenhausen, K. Mueller, M. H. Langston, B. Meister, and R. Lethin. 2016. An Interactive Visual Tool for Code Optimization and Parallelization Based on the Polyhedral Model. In 45th International Conference on Parallel Processing Workshops (ICPPW'16). 309-318. https://doi.org/10.1109/ICPPW.2016.52

[27] Gregor Richards, Ellen Arteca, and Alexi Turcotte. 2017. The VM Already Knew That: Leveraging Compile-time Knowledge to Optimize Gradual Typing. Proc. ACM Program. Lang. 1, OOPSLA, Article 55 (Oct. 2017), 27 pages.

[28] Gregor Richards, Francesco Zappa Nardelli, and Jan Vitek. 2015. Concrete Types for TypeScript. In 29th European Conference on ObjectOriented Programming, ECOOP 2015, July 5-10, 2015, Prague, Czech Republic. 76-100. https://doi.org/10.4230/LIPIcs.ECOOP.2015.76

[29] Richard Roberts, Stefan Marr, Michael Homer, and James Noble. 2017. Toward Virtual Machine Adaption Rather than Reimplementation. In MoreVMs'17: 1st International Workshop on Workshop on Modern Language Runtimes, Ecosystems, and VMs at <Programming $>$ 2017. Presentation. 
[30] Richard Roberts, Stefan Marr, Michael Homer, and James Noble. 2019. Transient Typechecks are (Almost) Free. In ECOOP.

[31] Jeremy G. Siek and Walid Taha. 2006. Gradual typing for functional languages. In Seventh Workshop on Scheme and Functional Programming, Vol. Technical Report TR-2006-06. University of Chicago, 81-92.

[32] Jeremy G. Siek and Walid Taha. 2007. Gradual Typing for Objects. In ECOOP 2007 - Object-Oriented Programming, 21st European Conference, Berlin, Germany, July 30 - August 3, 2007, Proceedings. 2-27.

[33] Jeremy G. Siek, Michael M. Vitousek, Matteo Cimini, and John Tang Boyland. 2015. Refined Criteria for Gradual Typing. In 1st Summit on Advances in Programming Languages (SNAPL 2015) (Leibniz International Proceedings in Informatics (LIPIcs)), Thomas Ball, Rastislav Bodik, Shriram Krishnamurthi, Benjamin S. Lerner, and Greg Morrisett (Eds.), Vol. 32. Schloss Dagstuhl-Leibniz-Zentrum fuer Informatik, 274-293.

[34] Jeremy G. Siek, Michael M. Vitousek, Matteo Cimini, Sam TobinHochstadt, and Ronald Garcia. 2015. Monotonic References for Efficient Gradual Typing. In European Symposium on Programming (ESOP). 432-456.

[35] Vincent St-Amour, Sam Tobin-Hochstadt, and Matthias Felleisen. 2012. Optimization Coaching: Optimizers Learn to Communicate with Programmers. In Proceedings of the ACM International Conference on Object Oriented Programming Systems Languages and Applications (OOPSLA'12). ACM, 163-178. https://doi.org/10.1145/2384616.2384629

[36] Asumu Takikawa, Daniel Feltey, Ben Greenman, Max S. New, Jan Vitek, and Matthias Felleisen. 2016. Is Sound Gradual Typing Dead? In Proceedings of the 43rd Annual ACM SIGPLAN-SIGACT Symposium on Principles of Programming Languages (POPL'16). ACM, 456-468.

[37] Michael M. Vitousek, Andrew M. Kent, Jeremy G. Siek, and Jim Baker. 2014. Design and evaluation of gradual typing for Python. In DLS'14, Proceedings of the 10th ACM Symposium on Dynamic Languages, part of SPLASH 2014, Portland, OR, USA, October 20-24, 2014. 45-56.

[38] Michael M. Vitousek, Jeremy G. Siek, and Avik Chaudhuri. 2019. Optimizing and Evaluating Transient Gradual Typing. CoRR abs/1902.07808 (2019). arXiv:1902.07808 http://arxiv.org/abs/1902.07808

[39] Michael M. Vitousek, Cameron Swords, and Jeremy G. Siek. 2017. Big Types in Little Runtime: Open-world Soundness and Collaborative Blame for Gradual Type Systems. In Proceedings of the 44th ACM SIGPLAN Symposium on Principles of Programming Languages (POPL'17). ACM, 762-774.

[40] Andreas Wöß, Christian Wirth, Daniele Bonetta, Chris Seaton, Christian Humer, and Hanspeter Mössenböck. 2014. An Object Storage Model for the Truffle Language Implementation Framework. In Proceedings of the 2014 International Conference on Principles and Practices of Programming on the fava Platform: Virtual Machines, Languages, and Tools (PPP''14). ACM, 133-144.

[41] Thomas Würthinger, Christian Wimmer, Christian Humer, Andreas Wöß, Lukas Stadler, Chris Seaton, Gilles Duboscq, Doug Simon, and Matthias Grimmer. 2017. Practical Partial Evaluation for Highperformance Dynamic Language Runtimes. In Proceedings of the 38th ACM SIGPLAN Conference on Programming Language Design and Implementation (PLDI'17). ACM, 662-676.

[42] Thomas Würthinger, Christian Wimmer, Andreas Wöß, Lukas Stadler, Gilles Duboscq, Christian Humer, Gregor Richards, Doug Simon, and Mario Wolczko. 2013. One VM to Rule Them All. In Proceedings of the 2013 ACM International Symposium on New Ideas, New Paradigms, and Reflections on Programming \& Software (Onward! 2013). ACM, 187-204.

[43] Thomas Würthinger, Andreas Wöß, Lukas Stadler, Gilles Duboscq, Doug Simon, and Christian Wimmer. 2012. Self-Optimizing AST Interpreters. In Proceedings of the 8th Dynamic Languages Symposium (DLS'12). 73-82. https://doi.org/10.1145/2384577.2384587 